\title{
Torta de mamona não destoxificada na indução da muda forçada em poedeiras comerciais
}

\author{
[Non-detoxified castor bean for molt induction in laying hens] \\ K.S.M. Mucida ${ }^{1}$, E.R. Freitas ${ }^{2}$, C.B. Miranda ${ }^{3}$, G.A.J. Nascimento ${ }^{2}$, J.S.C. Lima ${ }^{3}$ \\ ${ }^{1}$ Aluna de pós-graduação - Universidade Federal do Ceará - Fortaleza, CE \\ ${ }^{2}$ Universidade Federal do Ceará - Fortaleza, CE \\ ${ }^{3}$ Aluno de graduação - Universidade Federal do Ceará - Fortaleza, CE
}

\begin{abstract}
RESUMO
Avaliou-se o uso de dietas com diferentes porcentagens de torta de mamona não destoxificada (TM) na indução da muda forçada, sendo utilizadas 120 poedeiras Lohman LSL de 81 semanas, distribuídas ao acaso em quatro tratamentos, com cinco repetições de seis aves. Um dos tratamentos consistiu na indução da muda pelo método do jejum por 11 dias, e os demais no uso de dietas de muda, compostas pela mistura de dieta de postura e TM nas quantidades de 20,30 e $40 \%$, por até 21 dias ou até quando as aves atingissem $23 \%$ de perda do peso. Diferenças significativas foram observadas nos eritrócitos, no hematócrito, na concentração média de hemoglobina globular, na proteína total do plasma, nos leucócitos e na alanina aminotransferase, medidos durante a indução da muda, bem como no desempenho das aves após a muda, no que diz respeito ao consumo de ração, à porcentagem de postura, ao peso do ovo, à massa do ovo e à conversão alimentar. A qualidade dos ovos não variou estatisticamente entre os métodos avaliados. A utilização da dieta de muda forçada contendo $40 \%$ de TM promoveu resultados semelhantes aos obtidos com o método do jejum, tanto para consumo de ração, porcentagem de postura, peso do ovo, massa do ovo, e conversão alimentar, como inerentes à qualidade dos ovos, quanto para densidade específica, unidade Haugh, porcentagens de gema, casca e albúmen. No entanto, menores alterações nos valores de eritrócitos, hematócrito e alanina aminotransferase foram observadas nesse método supracitado. O uso da dieta de muda contendo $40 \%$ de TM mostrou-se uma alternativa viável ao uso do método do jejum.
\end{abstract}

Palavras-chave: hematologia, muda forçada, qualidade dos ovos

\begin{abstract}
We evaluated diets with different percentages of non-detoxified castor bean (TM) in the induction of molt, with 120 Lohman LSL hens at 81 weeks of age being used, randomly allotted to four treatments with five replicates of six birds each. One of the treatments consisted in the induction of changes by the method of fasting for 11 days, and the others used diet switches, composed by mixing posture and TM diet in the amounts of 20, 30 and $40 \%$ for up to 21 days or until when the birds reach $23 \%$ weight loss. Significant differences were observed in erythrocytes, hematocrit, mean concentration of corpuscular hemoglobin, total protein in plasma, leukocytes and alanine aminotransferase measured during induction of changes, and the performance of birds after moulting, such as feed intake, percentage of laying, egg weight, egg mass and feed conversion. The quality of the eggs did not vary significantly among the methods evaluated. The use of forced molting diet containing 40\% of TM promoted results similar to those obtained with the method of fasting, both feed intake and the percentage of egg, egg weight, egg mass, and feed conversion, as inherent quality of eggs, as the specific gravity, Haugh unit, yolk percentage, albumen and shell. However, minor changes in the values of erythrocytes, hematocrit and alanine aminotransferase were observed in the method above. The use of diets containing 40\% change TM proved to be a viable alternative to the use of the fasting method.
\end{abstract}

Keywords: hematology, forced molting, egg quality

Recebido em 30 de outubro de 2012

Aceito em 14 de dezembro de 2013

E-mail: magalhaesks@uol.com.br 


\section{INTRODUÇÃO}

A muda forçada é uma prática de manejo muito utilizada na indústria avícola produtora de ovos, e sua aplicação somente é justificada quando resulta em beneficio econômico ao produtor. Entretanto, a tomada de decisão depende de numerosos fatores, principalmente dos custos com formação de frangas de reposição, da produção do lote, da qualidade dos ovos, da situação de oferta e dos preços de ovos no mercado consumidor, da máxima utilização das instalações e do método de muda empregado.

A restrição total de alimento para a redução de aproximadamente $30 \%$ do peso corporal tem sido a técnica mais utilizada para muda forçada em poedeiras comerciais, sobretudo por ser de fácil aplicação e menos onerosa e por apresentar resultados satisfatórios após o processo de muda (Souza et al., 2010). No entanto, a utilização do método de jejum para induzir a muda em aves tem sido questionada em todo o mundo por ser uma prática antagônica ao bem-estar das aves que favorece uma incidência maior de problemas infecciosos nas aves (Landers et al., 2005; Khoshoei e Khajali, 2006).

As pesquisas com métodos de indução à muda forçada que não submetam as aves ao jejum têm sido incentivadas. Entre as alternativas para indução da muda forçada em poedeiras comerciais, pode-se destacar a utilização de rações de muda contendo alimentos ricos em fibra e ou com a presença de fatores antinutricionais, como a jojoba (Vermaut et al., 1998), a semente e o farelo de algodão (Davis et al., 2002; Khoshoei e Khajali, 2006), o farelo de trigo (Biggs et al., 2004; Khoshoei e Khajali, 2006; Koelkebeck et al., 2006), o feno de alfafa (Donalson et al., 2005; Landers et al., 2005) e a casca de arroz (Souza et al., 2010). Entretanto, segundo Landers et al. (2005), um método de indução de muda forçada será aceito pela indústria avícola se este produzir o estímulo necessário para a muda, garantindo regressão suficiente do sistema reprodutor, da produção e qualidade dos ovos no período após a muda, semelhantemente ao resultado obtido com o método do jejum.

A mamona (Ricinus communis L.) é uma xerófila de origem afroasiática, tolerante à escassez de água e bastante utilizada na produção de biocombustíveis. Gera subprodutos como a torta, o farelo e a casca, que podem ser utilizados na alimentação animal após destoxificação.

Na torta não destoxificada, além do elevado nível de fibra, podem ser encontradas três substâncias tóxicas: ricina, ricinina e o $\mathrm{CB}-1 \mathrm{~A}$, que são classificados como uma proteína, um alcaloide e um complexo alergênico, respectivamente. A ricina é a principal responsável pela toxidez da torta de mamona. Dependendo da dose e do tempo de ingestão, os animais se recuperam dos sintomas de intoxicação em dois a seis dias (Bandeira et al., 2004). A presença desses elementos tóxicos torna a mamona imprópria para o consumo animal; sua utilização somente é permitida se passar por um processo de desintoxicação, que, muitas vezes, é complexo e caro (Pina et al., 2005).

O objetivo deste experimento foi avaliar a utilização de dietas contendo diferentes porcentagens de torta de mamona não destoxificada (TM) na indução da muda forçada, bem como seus efeitos sobre as variáveis sanguíneas das aves, a produção, o peso e a qualidade dos ovos no período pós-muda, comparando os resultados com aqueles obtidos com aves que foram induzidas à muda forçada por meio do método de jejum.

\section{MATERIAL E MÉTODOS}

Para realização deste ensaio, foram utilizadas 120 poedeiras brancas da linhagem comercial Lohman LSL-Clássica, com 81 semanas de idade. As aves foram selecionadas, pesadas e, com base no peso de $1580 \pm 120 \mathrm{~g}$, distribuídas em delineamento inteiramente ao acaso, com quatro tratamentos e cinco repetições com seis aves por unidade experimental. As aves foram alojadas em aviário de produção, equipado com gaiolas de arame galvanizado $(25 \times 40 \times 30 \mathrm{~cm})$, dispostas em duas fileiras duplas com um corredor de serviço. Cada gaiola tinha bebedouro tipo copo, comedouro tipo calha, coletor de ovos, e havia duas aves por gaiola.

Os tratamentos consistiram na indução da muda forçada pelo método do jejum (T1) e no uso de dietas de muda compostas pela mistura da ração de postura (Tab. 1) com a torta de mamona não destoxificada (TM) nas quantidades de 20, $30 \mathrm{e}$ $40 \%$ (T2, T3 e T4, respectivamente). 
A TM utilizada foi o subproduto obtido da extração mecânica (prensagem) do óleo da semente à temperatura de $70^{\circ} \mathrm{C}$, proveniente de uma pequena usina de extração de óleo, no estado do Ceará. A TM foi submetida à análise de composição química no laboratório de nutrição animal do Departamento de Zootecnia da Universidade Federal do Ceará (DZ/UFC) e apresentou valores de $92,2 \%$ de matéria seca, $31,1 \%$ de proteína bruta, $48,9 \%$ de fibra bruta, $46,88 \%$ de fibra em detergente neutro e 40,7 de fibra em detergente ácido, com base na matéria seca.

$\mathrm{Na}$ formulação das dietas, foram considerados os valores e a composição dos alimentos propostos por Rostagno et al. (2005) e os níveis nutricionais propostos no manual da linhagem Lohmann LSL-Clássica (2009). As composições percentuais e calculadas das rações experimentais podem ser observadas na Tab. 1 .

Tabela 1. Composição percentual e calculada das rações experimentais

\begin{tabular}{|c|c|c|}
\hline Ingrediente & Pré-postura & Postura \\
\hline Milho & 67,50 & 67,52 \\
\hline Farelo de soja & 19,23 & 21,17 \\
\hline Farelo de trigo & 5,44 & - \\
\hline Calcário calcítico & 5,44 & 9,24 \\
\hline Fosfato monobicálcico & 1,62 & 1,32 \\
\hline Sal comum & 0,42 & 0,37 \\
\hline Suplemento vitamínico ${ }^{1}$ & 0,20 & 0,20 \\
\hline Suplemento mineral ${ }^{2}$ & 0,05 & 0,05 \\
\hline DL - Metionina 99\% & 0,10 & 0,13 \\
\hline Total & 100,00 & 100,00 \\
\hline \multicolumn{3}{|c|}{ Composição nutricional calculada } \\
\hline $\mathrm{EM} \mathrm{kcal} / \mathrm{kg}$ & 2.750 & 2.800 \\
\hline Proteína bruta $(\%)$ & 15,50 & 15,50 \\
\hline Metionina + Cistina $(\%)$ & 0,62 & 0,65 \\
\hline Metionina $(\%)$ & 0,35 & 0,38 \\
\hline Lisina $(\%)$ & 0,74 & 0,75 \\
\hline Treonina $(\%)$ & 0,60 & 0,60 \\
\hline Triptofano (\%) & 0,18 & 0,18 \\
\hline Cálcio $(\%)$ & 2,50 & 3,90 \\
\hline Fósforo disponível (\%) & 0,42 & 0,35 \\
\hline Sódio $(\%)$ & 0,20 & 0,18 \\
\hline \multicolumn{3}{|c|}{ 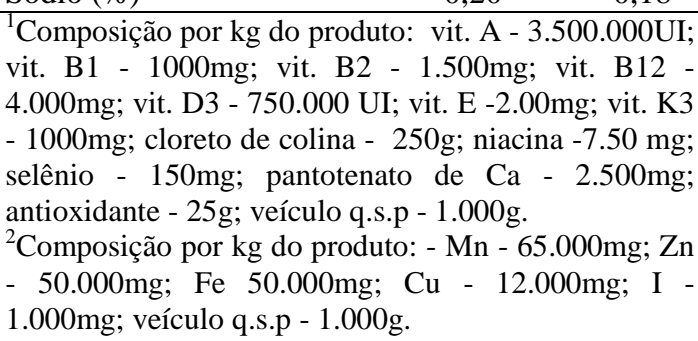 } \\
\hline
\end{tabular}

O método do jejum foi aplicado por 11 dias. Para os demais tratamentos, estabeleceu-se a suspensão da ração de muda quando as aves atingissem a média de $23 \%$ de perda de peso ou, no máximo, até 21 dias. Após o jejum (T1) e após as aves terem atingido uma perda de peso desejada de $23 \%$ ou terem completado 21 dias de tratamento (T2, T3 e T4), elas receberam dieta pré-postura (Tab. 1), em quantidades crescentes, até completarem 21 dias. Em todas as fases do experimento, as rações foram fornecidas à vontade. Durante o período de muda, a iluminação artificial foi suspensa, porém, a partir do $28^{\circ}$ dia, iniciou-se a implantação do programa de luz até o máximo de 16 horas de luz por dia.

Durante a indução da muda, foi registrado o tempo necessário para a parada da produção de ovos, a porcentagem de postura no período, bem como a porcentagem de perda de peso e o consumo médio de ração na primeira semana e ao final da aplicação de cada tratamento.

Amostras de sangue foram coletadas de uma ave por repetição ao final da indução da muda. O sangue foi coletado por punção da veia braquial, colocado em tubos contendo anticoagulante, ácido etilenodiaminotetracético (EDTA), e foi analisado no laboratório, a fim de se determinarem o número de eritrócitos e de hemoglobina, a porcentagem de hematócrito, o volume globular médio (VGM), a concentração média de hemoglobina globular (CHGM), a proteína total plasmática, o número de leucócitos, as enzimas aspartato aminotransferase (AST) e alanina aminotransferase (ALT).

O desempenho após a muda foi avaliado por 105 dias, divididos em cinco períodos de 21 dias. As variáveis avaliadas foram consumo de ração (g/ave/dia), produção de ovos (\%/ave/dia), massa de ovo (g/ave/dia) e conversão alimentar ( $\mathrm{kg} \mathrm{de}$ ração/kg de ovo).

A análise de qualidade dos ovos foi realizada uma vez por semana, durante todo o período experimental. Para isso, os ovos oriundos das aves de cada repetição foram coletados, e dois deles foram selecionados aleatoriamente, para se determinar inicialmente o peso e, em seguida, a gravidade específica (GE), utilizando-se os procedimentos descritos por Freitas et al. (2004). Foi montado sobre uma balança de precisão 
Marte $(0,01 \mathrm{~g})$ um sistema de pesagem dos ovos para obtenção do peso do ovo no ar e na água. Os valores do peso do ovo no ar e na água foram anotados para o cálculo da GE, com base na equação $\mathrm{GE}=\mathrm{PO} /(\mathrm{PA} \times \mathrm{F})$, em que: $\mathrm{PO}=$ peso do ovo no ar, $\mathrm{PA}=$ peso do ovo na água e $\mathrm{F}=$ fator de correção da temperatura.

Após as pesagens para determinação da GE, os ovos foram quebrados sobre uma superfície de vidro para determinação da altura do albúmen, com o uso de um micrômetro de profundidade. Os dados da altura do albúmen e do peso dos ovos foram utilizados no cálculo da unidade Haugh (UH), por meio da equação UH $=100 \log$ $\left(\mathrm{H}+7,57-1,7 \mathrm{~W}^{0,37}\right)$, em que: $\mathrm{H}=$ altura do albúmen $(\mathrm{mm})$ e $\mathrm{W}=$ peso do ovo $(\mathrm{g})$. Foi verificada também a porcentagem de gema, casca e albúmen.

Os dados foram submetidos à analise de variância utilizando-se o programa Statistical Analasys System (SAS, 2000), e a diferença entre médias foi detectada pelo teste SNK $(\mathrm{p}<0,05)$.

\section{RESULTADOS E DISCUSSÃO}

O aumento da participação da TM nas rações promoveu redução no consumo de ração até sete dias e durante o tratamento. Esse efeito pode ser atribuído à ação conjunta de fatores negativos da TM, tais como o elevado nível de fibra e os fatores tóxicos presentes nesse subproduto, que podem exercer efeitos antinutricionais (Tab. 2).

Tabela 2. Desempenho de poedeiras comerciais durante o período de indução à muda forçada

\begin{tabular}{|c|c|c|c|c|c|c|}
\hline \multirow[b]{2}{*}{ Parâmetros } & \multicolumn{4}{|c|}{ Tratamentos ${ }^{1}$} & \multirow[b]{2}{*}{ Média } & \multirow[b]{2}{*}{$\begin{array}{l}\mathrm{CV}^{2} \\
(\%)\end{array}$} \\
\hline & $\begin{array}{c}\mathrm{T} 1 \\
\text { Jejum }\end{array}$ & $\begin{array}{c}\mathrm{T} 2 \\
20 \% \mathrm{TM}\end{array}$ & $\begin{array}{c}\text { T3 } \\
30 \% \mathrm{TM}\end{array}$ & $\begin{array}{c}\mathrm{T} 4 \\
40 \% \mathrm{TM}\end{array}$ & & \\
\hline \multicolumn{7}{|l|}{ Consumo de ração (g/ave/dia) } \\
\hline Até 7 dias & $0,0 \mathrm{~d}$ & $30,93 a$ & $22,06 b$ & $16,35 c$ & 17,34 & 15,70 \\
\hline Durante o tratamento & $0,0 \mathrm{~d}$ & $39,20 \mathrm{a}$ & $26,23 b$ & $17,85 \mathrm{c}$ & 20,82 & 12,74 \\
\hline \multicolumn{7}{|l|}{ Perda de peso $(\%)$} \\
\hline Até 7 dias & $25,45 \mathrm{a}$ & $17,58 b$ & $19,35 b$ & $19,63 b$ & 20,50 & 8,23 \\
\hline Durante o tratamento & $30,88 \mathrm{a}$ & $18,82 \mathrm{c}$ & $24,90 \mathrm{~b}$ & $23,37 \mathrm{~b}$ & 24,50 & 8,24 \\
\hline $\begin{array}{l}\text { Tempo para parada } \\
\text { da postura (dias) }\end{array}$ & $4,2 \mathrm{~b}$ & $7,6 \mathrm{a}$ & $4,2 \mathrm{~b}$ & $5,8 \mathrm{ab}$ & 5,45 & 27,40 \\
\hline Duração do tratamento (dias) & $11 \mathrm{c}$ & $21 \mathrm{a}$ & $17 \mathrm{~b}$ & $11 \mathrm{c}$ & 15 & 0 \\
\hline $\begin{array}{l}\text { Produção de ovos } \\
\text { até } 21 \text { dias(\%) }\end{array}$ & $6,33 b$ & $13,00 \mathrm{a}$ & $6,83 b$ & $6,17 b$ & 8,08 & 29,87 \\
\hline
\end{tabular}

${ }^{1}$ T1-indução da muda pelo método do jejum; T2- dieta de muda forçada composta pela dieta de postura e $20 \%$ de torta de mamona não destoxificada (TM); T3- dieta de muda forçada composta pela dieta de postura e 30\% de TM; T4-dieta de muda forçada composta pela dieta de postura e $40 \%$ de TM. ${ }^{2} \mathrm{CV}$ - coeficiente de variação. Na linha, médias seguidas de letras distintas diferem significativamente pelo teste $\mathrm{SNK}(\mathrm{p}<0,05)$.

A perda de peso, após sete dias do início do período de indução da muda e durante a aplicação de cada método, foi maior para as aves submetidas ao jejum quando comparado com os demais tratamentos. Com o uso da dieta contendo TM, ocorreu perda de peso semelhante para as aves alimentadas com as diferentes quantidades de TM nas dietas até sete dias de aplicação dos métodos de indução à muda forçada. No entanto, como as aves alimentadas com dietas contendo $20 \%$ de TM não atingiram a perda de peso mínima de $23 \%$, a aplicação desse tratamento se prolongou até 21 dias.
A redução na velocidade de perda de peso, durante a indução da muda forçada, e, consequentemente, o aumento do tempo para que se atingisse o nível desejado para as aves submetidas à indução com uso de dietas de muda podem ser atribuídos ao maior consumo de ração com níveis mais baixos de participação da TM na dieta. Esses resultados se assemelham aos relatados por Souza et al. (2010), que avaliaram o uso de ração de muda composta por ração de postura e casca de arroz na proporção de 50 e $75 \%$ e verificaram que quanto maior a proporção de ração de postura na mistura, menor foi a perda 
de peso e maior o tempo para atingir a perda de peso de $25 \%$.

As aves submetidas à indução da muda pelo método do jejum e com o uso do TM em todos os níveis interromperam a produção de ovos. Entretanto, as aves que receberam $20 \%$ de TM suspenderam a produção de ovos mais tardiamente. Esse fato contribuiu para que essas aves apresentassem a maior porcentagem de postura, durante os 21 dias de aplicação dos métodos de indução.

O aumento no número de dias para a parada total da postura durante a indução da muda forçada tem sido relatado na literatura (Donalson et al., 2005; Landers et al., 2005; Souza et al., 2010) quando se compara o uso de métodos alternativos com o fornecimento de ração em relação ao método do jejum. Isso ocorre em razão de uma menor exaustão das reservas corporais das aves quando estas recebem algum tipo de alimentação durante essa prática. A parada total da postura e o nível da perda de peso corporal das aves no período de indução da muda são fatores importantes, pois podem influenciar o desempenho no período após a muda, obtendo-se melhor desempenho com parada total da postura o mais rápido possível e perda de peso entre 20 e $30 \%$ do peso inicial (Baker et al., 1983).
Para a análise sanguínea, houve diferença significativa entre os métodos de indução de muda para os valores de eritrócitos, hematócrito, CHGM, proteína total, leucócitos e ALT. Entretanto, os valores de hemoglobina, VGM e AST não variaram significativamente entre os métodos (Tab. 3).

As aves submetidas à indução de muda com dieta contendo $40 \%$ de TM apresentaram valores de eritrócitos e hematócrito significativamente menores em relação aos demais tratamentos que não diferiram entre si. A redução para esses parâmetros apresentada pelas aves submetidas ao uso de $40 \%$ de TM na dieta de muda em relação aos demais métodos pode ter ocorrido por uma associação entre a desnutrição e a intoxicação causada pela ingestão da TM. Segundo Schmidt et al. (2007), a redução de eritrócitos e hematócrito caracteriza anemia em aves, que pode decorrer, entre outros fatores, da desnutrição e de intoxicações.

Diferentemente do observado na presente pesquisa, Khajali et al. (2008) relataram semelhanças no hematócrito em aves submetidas à muda forçada com uso de rações de muda com substituição do milho por trigo com ou sem sal em relação ao método do jejum.

Tabela 3. Parâmetros sanguíneos de poedeiras comerciais submetidas à muda forçada

\begin{tabular}{|c|c|c|c|c|c|c|}
\hline \multirow[b]{2}{*}{ Variáveis ${ }^{1}$} & \multicolumn{4}{|c|}{ Tratamentos $^{2}$} & \multirow[b]{2}{*}{ Média } & \multirow[b]{2}{*}{$\begin{array}{c}\mathrm{CV}^{3} \\
(\%)\end{array}$} \\
\hline & $\begin{array}{c}\mathrm{T} 1 \\
\text { Jejum }\end{array}$ & $\begin{array}{c}\text { T2 } \\
20 \% \mathrm{TM}\end{array}$ & $\begin{array}{c}\text { T3 } \\
30 \% \mathrm{TM}\end{array}$ & $\begin{array}{c}\mathrm{T} 4 \\
40 \% \mathrm{TM}\end{array}$ & & \\
\hline $\begin{array}{l}\text { Eritrócitos } \\
\left(\mathrm{x} 10^{6} / \mathrm{mm}^{3}\right)\end{array}$ & $3,14 \mathrm{a}$ & $3,36 a$ & $3.32 \mathrm{a}$ & $2,21 \mathrm{~b}$ & 3,00 & 19,58 \\
\hline Hemoglobina (g/dL) & $13,90 \mathrm{a}$ & $14,14 \mathrm{a}$ & $14,56 \mathrm{a}$ & $14,18 \mathrm{a}$ & 14,19 & 12,17 \\
\hline Hematócrito $(\%)$ & $38,00 \mathrm{a}$ & $33,60 \mathrm{a}$ & $33,00 \mathrm{a}$ & $25,80 \mathrm{~b}$ & 32,70 & 13,09 \\
\hline $\operatorname{VGM}\left(\mathrm{mm}^{3}\right)$ & $112,70 \mathrm{a}$ & $108,50 \mathrm{a}$ & $97,34 \mathrm{a}$ & $120,52 \mathrm{a}$ & 109,76 & 13,42 \\
\hline CHGM $(\mathrm{g} / \mathrm{dL})$ & $37,00 \mathrm{c}$ & $42,00 \mathrm{~b}$ & $43,56 b$ & $50,12 \mathrm{a}$ & 43,17 & 6,40 \\
\hline $\begin{array}{l}\text { Proteína total do } \\
\text { plasma }(\mathrm{g} / \mathrm{dL})\end{array}$ & $5,48 \mathrm{a}$ & $5,20 \mathrm{ab}$ & $4,64 b$ & $5,24 \mathrm{ab}$ & 5,14 & 7,46 \\
\hline Leucócitos $\left(\mathrm{mm}^{3}\right)$ & $502 c$ & $1.620 \mathrm{a}$ & $1.180 \mathrm{~b}$ & $1.400 \mathrm{ab}$ & 1,17 & 22,28 \\
\hline $\mathrm{ALT}(\mathrm{U} / \mathrm{L})$ & $13,60 \mathrm{a}$ & $6,80 \mathrm{bc}$ & $5,00 \mathrm{c}$ & $9,00 \mathrm{~b}$ & 8,60 & 22,51 \\
\hline AST (U/L) & $234 \mathrm{a}$ & $234 \mathrm{a}$ & $214 a$ & $236 a$ & 229 & 27,00 \\
\hline
\end{tabular}

${ }^{1}$ VGM-volume globular médio; CHGM-concentração de hemoglobina corpuscular média; ALT-alanina aminotransferase; AST-aspartato aminotransferase. ${ }^{2}$ T1-indução da muda pelo método do jejum; T2- dieta de muda forçada composta pela dieta de postura e $20 \%$ de torta de mamona não destoxificada (TM); T3- dieta de muda forçada composta pela dieta de postura e 30\% de TM; T4-dieta de muda forçada composta pela dieta de postura e $40 \%$ de TM. ${ }^{3} \mathrm{CV}$-coeficiente de variação. Na linha, médias seguidas de letras distintas diferem significativamente pelo teste SNK $(\mathrm{p}<0,05)$. 
Os valores de CHGM das aves submetidas à muda pelo método do jejum foram significativamente menores em relação aos das aves dos demais tratamentos, e os valores para as aves submetidas a dietas contendo $20 \%$ e $30 \%$ de TM não diferiram entre si, porém foram menores que o das aves submetidas a dietas com $40 \%$ de TM.

Os resultados do CHGM das aves submetidas ao jejum evidenciam uma hipocromia do sangue dessas aves em comparação ao das aves alimentadas com $40 \%$ de TM na dieta de muda. Dessa forma, é importante ressaltar que, embora as células vermelhas do sangue das aves submetidas ao uso de $40 \%$ de TM tenham reduzido, a sua qualidade como transportadoras de oxigênio foi mantida, já que os maiores valores de CHGM foram determinados no sangue dessas aves.

Em relação à quantidade de proteína total no plasma sanguíneo, observou-se diferença significativa apenas entre os valores das aves submetidas ao jejum e das aves alimentadas com dieta contendo $30 \%$ de TM. Esse segundo método de indução à muda, no entanto, foi o que promoveu redução na proteína total do plasma.

Lesões hepáticas podem levar à diminuição da concentração de proteínas totais do plasma, pois o fígado é o órgão que sintetiza as proteínas, principalmente a albumina (Schimid et al., 2007). Assim, a redução dessas proteínas com o uso de TM pode ser associada aos efeitos tóxicos desse alimento. Isso fica evidente nas aves alimentadas com dietas contendo 20 e $30 \%$ de $\mathrm{TM}$, as quais apresentaram os menores resultados, já que essas aves foram as que consumiram a ração contendo TM por um maior período de tempo, 21 e 17 dias, respectivamente.

A quantidade de leucócitos das aves submetidas ao método do jejum foi inferior a das aves alimentadas com as dietas contendo TM nas três quantidades estudadas $(20,30$ e 40\%). Entretanto, entre as aves alimentadas com dietas contendo os diferentes níveis de mamona, observou-se que o número de leucócitos diferiu significativamente apenas entre as aves alimentadas com 20 ou $30 \%$ de TM.

Segundo Schimidt et al. (2007), a leucopenia pode ocorrer por infecções sistêmicas agudas ou estresse severo. Assim, conforme os resultados obtidos, a condição de estresse severo causado pelo jejum diminui a quantidade de células brancas de defesa no sangue em relação às aves alimentadas com TM. McReynolds et al. (2009) avaliaram a influência dos métodos de muda forçada sobre os leucócitos das poedeiras e verificaram valores significativamente menores de leucócitos para aves submetidas ao método de jejum, enquanto os valores para aves alimentadas com alfafa não diferiram entre as aves alimentadas com ração de muda.

Os valores de ALT das aves submetidas à muda pelo método do jejum foram significativamente maiores em relação aos das aves dos demais tratamentos. Os valores para as aves submetidas a $20 \%$ e $30 \%$ de TM não diferiram entre si, e apenas os resultados obtidos com $30 \%$ foram significativamente menores que o das aves submetidas a $40 \%$ de TM.

De acordo com Schimidt et al. (2007), as enzimas hepáticas AST e ALT extravasam para o sangue quando ocorrem lesões no fígado, aumentando as suas concentrações. Entretanto, conforme esses autores, como a ALT é encontrada tanto no citosol do hepatócito como nas células dos músculos e de outros tecidos, a sua determinação para caracterizar lesão hepática nas aves tem valor limitado. Dessa forma, o valor significativamente maior de ALT nas aves submetidas à muda pelo método do jejum pode ser associado ao extravasamento da ALT muscular devido à maior perda de peso dessas aves no período de indução de muda, que chegou a aproximadamente $31 \%$ do peso vivo. Essa possibilidade também justifica as diferenças entre os métodos contendo TM nas dietas, cuja perda de peso foi da ordem de 18,8; 24,9 e $23,4 \%$ para o uso de ração de muda contendo 20 , 30 e $40 \%$ de TM, respectivamente.

Por outro lado, a ausência de diferenças significativas nos valores de AST e o aumento dos valores de ALT para as aves alimentadas com TM de mamona evidenciaram a ausência de uma ação tóxica severa da ricina sobre o fígado das aves. Esses resultados diferem dos relatos da literatura, que indicam danos hepáticos associados aos efeitos tóxicos da ricina presente na torta de mamona não destoxificada oferecida aos animais (Bandeira et al., 2004). Segundo dados relacionados por Távora (1982), as aves 
apresentam a maior tolerância à toxidade da mamona entre os animais, sendo a dose letal de ingestão da semente cerca de $14 \mathrm{mg} / \mathrm{kg}$ de peso vivo.
Conforme os resultados de desempenho após a muda, o consumo de ração, a porcentagem de postura, o peso e a massa de ovo, bem como a conversão alimentar, variaram significativamente entre as aves submetidas aos diferentes métodos de indução da muda (Tab. 4).

Tabela 4. Efeito das metodologias de indução à muda forçada sobre o desempenho de poedeiras comerciais no período após a muda

\begin{tabular}{|c|c|c|c|c|c|c|}
\hline \multirow[b]{2}{*}{ Variáveis } & \multicolumn{4}{|c|}{ Tratamentos $^{1}$} & \multirow[b]{2}{*}{ Média } & \multirow{2}{*}{$\begin{array}{l}\mathrm{CV}^{2} \\
(\%)\end{array}$} \\
\hline & $\begin{array}{c}\text { T1 } \\
\text { Jejum }\end{array}$ & $\begin{array}{c}\mathrm{T} 2 \\
20 \% \mathrm{TM}\end{array}$ & $\begin{array}{c}\text { T3 } \\
30 \% \text { TM }\end{array}$ & $\begin{array}{c}\mathrm{T} 4 \\
40 \% \mathrm{TM}\end{array}$ & & \\
\hline Consumo de ração (g/ave/dia) & $101,75 a$ & $94,64 b$ & $91,44 b$ & $98,24 a$ & 76,33 & 5,90 \\
\hline Porcentagem de postura (ave/dia) & $81,79 \mathrm{a}$ & $69,54 b$ & $72,34 b$ & $79,46 a$ & 96,89 & 4,13 \\
\hline Peso do ovo $(\mathrm{g})$ & $67,58 \mathrm{a}$ & $61,37 b$ & $61,83 b$ & $67,03 a$ & 64,77 & 2,50 \\
\hline Massa de ovo (g/ave/dia) & $55,22 \mathrm{a}$ & $42,65 b$ & $44,72 b$ & $53,19 \mathrm{a}$ & 49,56 & 6,00 \\
\hline Conversão alimentar $(\mathrm{kg} / \mathrm{kg})$ & $1,89 \mathrm{c}$ & $2,22 \mathrm{a}$ & $2,05 \mathrm{~b}$ & $1,85 \mathrm{c}$ & 1,98 & 5,37 \\
\hline
\end{tabular}

${ }^{1}$ T1-indução da muda pelo método do jejum; T2- dieta de muda forçada composta pela dieta de postura e $20 \%$ de torta de mamona não destoxificada (TM); T3- dieta de muda forçada composta pela dieta de postura e 30\% de TM; T4-dieta de muda forçada composta pela dieta de postura e $40 \%$ de TM. ${ }^{2} \mathrm{CV}$-coeficiente de variação. Na linha, médias seguidas de letras distintas diferem significativamente pelo teste SNK $(\mathrm{p}<0,05)$.

As aves submetidas à muda forçada com o uso de ração de muda contendo $40 \%$ de TM apresentaram valores estatisticamente semelhantes para consumo de ração, porcentagem de postura, peso e massa de ovo, bem como para conversão alimentar, em relação aos valores obtidos para as aves submetidas ao método do jejum $(p>0,05)$. Entretanto, as aves submetidas à ração de muda contendo $20 \%$ e $30 \%$ de TM apresentaram menor consumo, porcentagem de postura, peso e massa de ovo, e pior conversão alimentar $(\mathrm{p}<0,05)$ que as submetidas ao método do jejum e ao uso de ração contendo $40 \%$ de TM.

O aumento no consumo de ração após a muda forçada tem sido associado ao nível de perda de peso das aves durante a indução da muda, de modo que as aves que perderam mais peso tendem a apresentar maior ingestão de ração, na tentativa de recuperar o peso corporal, o que aconteceu no experimento.

Por outro lado, o pior desempenho das aves submetidas aos níveis mais baixos de TM nas dietas pode ser atribuído à menor perda de peso dessas aves durante a indução da muda, pois, como relatado anteriormente, o nível da perda de peso corporal das aves no período de indução da muda é um fator para otimizar o desempenho no período após a muda, obtendo-se melhor resultado com perda de 25 a $30 \%$ do peso no início da indução da muda forçada (Baker et al., 1983).

Fato semelhante foi relato por Donalson et al. (2005), que avaliaram o uso do feno de alfafa na indução da muda em poedeiras e observaram que as aves submetidas à ração contendo $70 \%$ de feno de alfafa tiveram menor produção de ovos no período após a muda em relação às submetidas ao método do jejum e ao uso de rações de muda contendo 100 e $90 \%$ de feno de alfafa. Para os autores, os resultados obtidos para essas aves podem ser relacionados a uma muda incompleta, visto que as aves submetidas a esse tratamento foram as que perderam menos peso durante a indução da muda e, também, porque existe uma relação direta entre a perda de peso corporal e a regressão dos órgãos do sistema reprodutor.

Com relação à qualidade dos ovos, observou-se que não houve diferença significativa entre os métodos de indução à muda forçada sobre os parâmetros densidade específica, unidade Haugh e porcentagens de gema, casca e albúmen (Tab. 5). 
Os resultados obtidos na presente pesquisa se assemelham aos da literatura, em que são encontrados relatos de semelhança na qualidade dos ovos, no período após a muda, entre a indução da muda pelo método do jejum e o uso de rações de muda contendo jojoba (Vermaut et al., 1998), semente e farelo de algodão (Davis $e t$ al., 2002; Khoshoei e Khajali, 2006), farelo de trigo (Biggs, et al., 2004; Khoshoei e Khajali, 2006; Koelkebeck et al., 2006); feno de alfafa (Donalson et al., 2005; Landers et al., 2005) e outros alimentos (Khoshoei e Khajali, 2006).

Tabela 5. Efeito das metodologias de indução à muda forçada sobre o peso e a qualidade dos ovos no período após a muda

\begin{tabular}{|c|c|c|c|c|c|c|}
\hline \multirow[b]{2}{*}{ Variáveis } & \multicolumn{4}{|c|}{ Tratamentos $^{1}$} & \multirow[b]{2}{*}{ Média } & \multirow{2}{*}{$\begin{array}{l}C^{2} \\
(\%)\end{array}$} \\
\hline & $\begin{array}{c}\text { T1 } \\
\text { Jejum }\end{array}$ & $\begin{array}{c}\mathrm{T} 2 \\
20 \% \mathrm{TM}\end{array}$ & $\begin{array}{c}\text { T3 } \\
30 \% \text { TM }\end{array}$ & $\begin{array}{c}\mathrm{T} 4 \\
40 \% \mathrm{TM}\end{array}$ & & \\
\hline Densidade específica & $1,0782 \mathrm{a}$ & $1,0770 \mathrm{a}$ & $1,0792 \mathrm{a}$ & $1,0826 a$ & 1,080 & 0,32 \\
\hline Unidade Haugh & $90,11 \mathrm{a}$ & $91,76 a$ & $92,32 \mathrm{a}$ & $90,83 \mathrm{a}$ & 91,26 & 2,54 \\
\hline Gema $(\%)$ & $23,29 a$ & $23,40 \mathrm{a}$ & $23,42 \mathrm{a}$ & $23,18 \mathrm{a}$ & 23,32 & 3,58 \\
\hline Casca $(\%)$ & $8,55 \mathrm{a}$ & $8,60 \mathrm{a}$ & $8,59 \mathrm{a}$ & $8,53 \mathrm{a}$ & 8,57 & 2,14 \\
\hline Albúmen (\%) & $61,50 \mathrm{a}$ & $61,33 a$ & $61,33 a$ & $6163 a$ & 61,45 & 1,50 \\
\hline
\end{tabular}

${ }^{\mathrm{T}}$ T1-indução da muda pelo método do jejum; T2- dieta de muda forçada composta pela dieta de postura e $20 \%$ de torta de mamona não destoxificada (TM); T3- dieta de muda forçada composta pela dieta de postura e 30\% de TM; T4-dieta de muda forçada composta pela dieta de postura e $40 \%$ de TM. ${ }^{2} \mathrm{CV}$-coeficiente de variação. Na linha, médias seguidas de letras distintas diferem significativamente pelo teste $\mathrm{SNK}(\mathrm{p}<0,05)$.

Diante das diversas possibilidades de alternativas de indução da muda forçada com a não privação das aves de alimento, Landers et al. (2005) consideraram que um determinado método alternativo de indução da muda forçada será aceito pela indústria avícola desde que produza o estímulo necessário para a muda, garantindo produção e qualidade dos ovos, no período após a muda, semelhantemente aos resultados obtidos com o método do jejum. Dessa forma, o uso de uma dieta de muda composta por ração de postura e torta de mamona não destoxificada em nível de $40 \%$ constitui um método alternativo para aplicação na avicultura de postura.

\section{CONCLUSÕES}

O uso de ração composta por ração de postura e torta de mamona não destoxificada em nível de $40 \%$ é uma alternativa viável ao método do jejum para indução da muda forçada em poedeiras Lohmann LSL-Clássica.

\section{REFERÊNCIAS}

BAKER, M.; BRAKE, J.; MCDANIEL, G.R. The relationship between body weight loss during an induced molt and postmolt egg production, egg weight and shell quality in caged layers. Poultry Sci., v.62, p.409-413, 1983.

BANDEIRA, D.A.; CARTAXO, W.V.; BELTRÃO, N.E.M. et al. Resíduos industrial da mamona como fonte alternativa na alimentação animal. In: CONGRESSO BRASILEIRO DE MAMONA, 2004, Campina Grande. Anais... Campina Grande: Embrapa Algodão, 2004.

BIGGS, P.E.; PERSIA, M.E.; KOELKEBECK, K.W.; PARSONS, C.M. Further evolution of non feed removal methods for molting programs. Poultry Sci., v.83, p.754-752, 2004.

DAVIS, A.J.; LORDELO, M.M.; DALE, N. Use of cottonseed meats in molting programs. $J$. Appl. Poultry Res., v.11, p.175-178, 2002.

DONALSON, L.M.; KIM, W.K.; HERERRA, P. et al. Utilizing different ratios of alfalfa and layer ration for molt induction and performance in commercial laying hens. Poultry Sci., v.84, p.362-369, 2005. 
FREITAS, E.R.; SAKOMURA, N.K.; GONZALEZ, M.M.; BARBOSA, N.A.A. Comparação de métodos de determinação da gravidade específica de ovos de poedeiras comerciais. Pesq. Agrop. Bras., v.39, p.509-512, 2004.

KHAJALI F.; KARIMI S.; AKHARI M. R. Physiological Response and Postmolt Performance of Laying Hens Molted by NonFeed Removal Methods. Amer. J. Anim. Veter. Sci., 13-17, 2008

KHOSHOEI, E.A.; KHAJALI, F. Alternative induced-molting methods for continuous feed withdrawal and their influence on post molt performance of laying hens. Intern. J. Poultry Sci., v.5, p.47-50, 2006.

KOELKEBECK, K.W.; PARSONS, C.M.; BIGGS, P.; UTTERBACK, P. Non withdrawal molting programs. J. Appl. Poultry Res., v.15, p.483-491, 2006.

LANDERS, K.L.; WOODWARD, C.L.; LI, X. et al. Alfalfa as a single dietary source for molt induction in laying hens. Biores. Tech., v.96, p.565-570. 2005.

LOHMANN LSL-CLÁSSICA. Guia de manejo da linhagem Lohmann LSL. Lohmann do Brasil: São José do Rio Preto/SP, 2009.

McREYNOLDS, J.L.; GENOVESE, K.J.; HE, $\mathrm{H}$. et al. Alfalfa as a nutritive modulator in maintaining the innate immune response during the molting process. Poultry Sci., v.18, p.410417, 2009
PINA, M.; SEVERINO, L.S.; BELTRÃO, N.E.M. et al. Novas alternativas de valorização para dinamizar a cultura da mamona no Brasil. Cadernos de Ciência \& Tecnologia, Brasília, v.22, p.453-462, 2005.

ROSTAGNO, H.S.; ALBINO, L.F.T.; DONZELE, J.L. et al. Tabelas brasileiras para aves e suínos: composição de alimentos e exigências nutricionais. Viçosa: UFV, 2005. $186 \mathrm{p}$.

STATISTICAL Analyses System - SAS: User's guide. Cary, 2000. CD ROM.

SCHMIDT, E.M.S.; LOCATELI-DITRICH, R.; SANTIN, E.; PAULILLO, A.C. Patologia clínica em aves de produção - Uma ferramenta para monitorar a sanidade avícola - Revisão. Arch. Vet. Sci., v.12, p.9-20, 2007.

SOUZA, K.M.; CARRIJO, A.S.; ALLAMAN, I.B. et al. Métodos alternativos de restrição alimentar na muda forçada de poedeiras comerciais. Rev. Bras. Zootec., v.39, p.356-362, 2010.

TÁVORA, F.J.A.F. A cultura da mamona. Fortaleza: Empresa de Pesquisa Agropecuária do Ceará (EPACE), 1982. 111p.

VERMAUT, S.; CONINCK, K.; ONAGBESAN, O. A jojoba-rich diet as a new forced molting method in poultry. J. Appl. Poultry Res., v.7, p.239-246, 1998. 